\title{
A Comparison of Nutritional Values, Bioactive Compounds, Amino Acids, and Antioxidant Activities of Alfalfa (Medicago sativa) Plant and Pellet for Use as Beneficial Material Ruminant Feed
}

\section{Supreena SRISAIKHAM}

Faculty of Agricultural Technology, Burapha University, Sa Kaeo Campus, Sa Kaeo 27160, Thailand

(Corresponding author's e-mail: supreena.sr@buu.ac.th)

Received: 21 May 2020, Revised: 25 September 2020, Accepted: 26 October 2020

\begin{abstract}
Aqueous extracts of whole alfalfa plants (AP) and alfalfa pellets (APe) were examined for nutritional values, bioactive compounds (total phenolic content (TPC), phenolic compounds (PC), total flavonoids content (TFC), flavonoids compounds (FC), isoflavones), amino acids (AA) and antioxidant activity of AP and APe assessed via a DHHP radical scavenging assay and ferric reducing antioxidant power (FRAP) assay. The potential ingredients in the form of AP and APe were used to evaluate the nutritional values measured by chemical composition, and to investigate bioactive compounds, AA and antioxidant activity to further contribute to use as a feed ingredient or application as a high-value alternative feed supplement in the ruminant diet. Crude protein and tannins were higher in the APe (18.17 and $3.08 \%$ ) than the AP (16.21 and $1.80 \%)$. Higher content of organic matter, crude fiber, neutraldetergent fiber, acid-detergent fiber, acid-detergent lignin was observed for AP than APe. The TPC and TFC were more abundant in AP than in APe and AP also showed stronger antioxidant activity via DPPH than APe, with positive correlations of TPC, TFC and DPPH. The highest values of gallic acid in terms of $\mathrm{PC}$ and apigenin in terms of FC of APe were 215.30 and $2278.27 \mu \mathrm{g} / \mathrm{g}$ dry weight (DW), respectively, while the highest levels of genistein and daidzein occurred in AP at 104.37 and $130.91 \mu \mathrm{g} / \mathrm{g} \mathrm{DW}$, respectively. The total of essential AA was found to be in vary the range of 9.33 to $16689.47 \mu \mathrm{g} / \mathrm{g} \mathrm{DW}$ in different forms of samples, with valine as the highest $(5451 \mu \mathrm{g} / \mathrm{g} \mathrm{DW})$ in APe, followed by phenylalanine $(4.30 \mu \mathrm{g} / \mathrm{g} \mathrm{DW})$ in AP.
\end{abstract}

Keywords: Alfalfa, Bioactive compounds, Amino acids, Antioxidant activity, Nutritional values

\section{Introduction}

Nowadays, a diet that focuses on a plant-based diet (vegetables, legumes, and grains) is now popular as people are more concerned about their health and they realize that meat or milk from livestock farming inevitably affects their health particularly concerning nutrition and food safety. In recent decades, phytochemical compounds, such as polyphenols, phenolic, flavonoids, and phytoestrogen derived from legumes, especially soybean or white kidney bean, have been widely researched as an alternative natural functional food supplement in terms of bioactive compounds. Although phytochemicals are not essential nutrients for a healthy life, they are beneficial for health due to their preventive properties and the reduction of the risk of non-communicable diseases (NCDs) [1]. Alfalfa (Medicago sativa L.) is recognized as one of the most important forage legumes because it is a major source of protein for livestock (dairy cattle, beef cattle, horses, sheep, goats, and other classes of domestic animals). Although the nutritional value of alfalfa and its phytochemicals is considerable and it is in increasing demand, it is still not popular in Thailand because of its high price and the low yield of some varieties [2]. Whereas, use of alfalfa in form of pellets on farms is more convenient [2]. Alfalfa produces more protein for the 
http://wjst.wu.ac.th

same area than sorghum in several countries of Europe and America. Furthermore, it is used in form of hay, silage, cubes, and fodder for pasture animals, and it includes compounds found including saponin, flavonoid, condensed tannin, coumestrol, carotenoid, and tocol [3]. Its tannin content can improve protein utilization through increased absorption of the essential AA from the small intestine in sheep fed Lotus corniculatus [4]. The important substances of flavonoids, isoflavones, flavonones, and chalcones are found in legumes, which are grouped of phytoestrogen, especially isoflavones are a sub-class of flavonoids [5], are an important substance found in soybeans and soybean products [6] and it is a phenolic found in food and plants. Natural flavonoids have similar properties to estrogen because their structure consists of a phenolic ring which makes them able to bind to the receptor protein of estrogen, so they are classified as phytoestrogens. Flavonoids can replace a lack or deficiency of estrogen in postmenopausal women and they can also be substituted for natural legume substances from isoflavones which could reduce the risk of other side effects from long-term synthetic or replacement hormones. Isoflavones also may reduce prostate cancer and osteoporosis risk [5]. The ingredients of ruminant animal feed contain soy, soybean meal, or soy products that derive from legumes and which provide high-quality proteins. It affects the concentration of isoflavone in soy (Glycine max (L.) Merrill) which contains daidzein and genistein, which are the isoflavones [6] and phytochemicals that are good for health, and phytoestrogen acts like a female hormone (Estrogen, 17b-estradiol E2 Isoflavones). Estrogen, 17b-estradiol E2 Isoflavones are derived from soybean and have been widely researched as an alternative natural functional food supplement to replace the estrogen hormone in women entering menopause. Although there have been some reports of alfalfa nutritional values, little is known about the bioactive compound, AA, and antioxidant activity information of different forms both in the AP cultivated in Thailand and APe.

The objective of this research was to compare nutritional values and identify and quantify the bioactive compounds and AA, as well as to investigate the antioxidant activities of AP and APe to obtain information regarding the application of alfalfa legume as a high-value ruminant feed ingredient material or supplement in Thailand.

\section{Materials and methods}

\section{Planting and harvesting alfalfa materials}

The alfalfa ( Medicago sativa) seeds were obtained from the Bureau of Animal Nutrition Development, Department of Livestock Development, Ministry of Agriculture and Cooperatives, Thailand. Alfalfa seeds were cultivated to be firmly grown in a seedling tray for 30 days, then the seedlings were transplanted into three field plots $\left(3 \times 3 \mathrm{~m}^{2}\right)$ at the experimental farm of the Agricultural Research Facility of the Faculty of Agricultural Technology, Burapha University ( BUU), Sa Kaeo campus, Watthananakhon district, Sa Kaeo, Thailand (Figure 1). The field plots are dry and shallow sandy soil with low fertility, which can be classified into groups of 49 types. After transplanting the seedlings, they were watered every 4 days and a chemical fertilizer formula 15-15-15 was added at the rate of $125 \mathrm{~kg}$ per hectare (ha). The AP were later harvested at cutting intervals of 75 days with the $3^{\text {rd }}$ cut (225 days) under the finished trial being partially funded (Grant no. 24/2562) [2]. The fresh AP samples were collected from each plot as whole plants at $10 \mathrm{~cm}$ above soil and then cut with a knife into 3-4 cm lengths. Pre-drying AP was dried at $60^{\circ} \mathrm{C}$ for at least $48 \mathrm{~h}$ in a hot air-oven and later ground through a 1 $\mathrm{mm}$ screen into a fine powder using a grinder, and then divided into 2 subsamples. One portion was used for determining chemical compositions, tannins and the other was extracted and used to determine bioactive compounds, AA, and antioxidant activity. These samples were all ground and stored pending analysis at $4{ }^{\circ} \mathrm{C}$ which was carried out immediately to avoid any changes in quality in the plastic lock bags.

\section{Collection of alfalfa pellets material}

The APe was dehydrated pellets that were used for comparison with the AP. The APe were thoroughly dried, ground, and stored by using the same procedure as for pre-drying AP previously. 


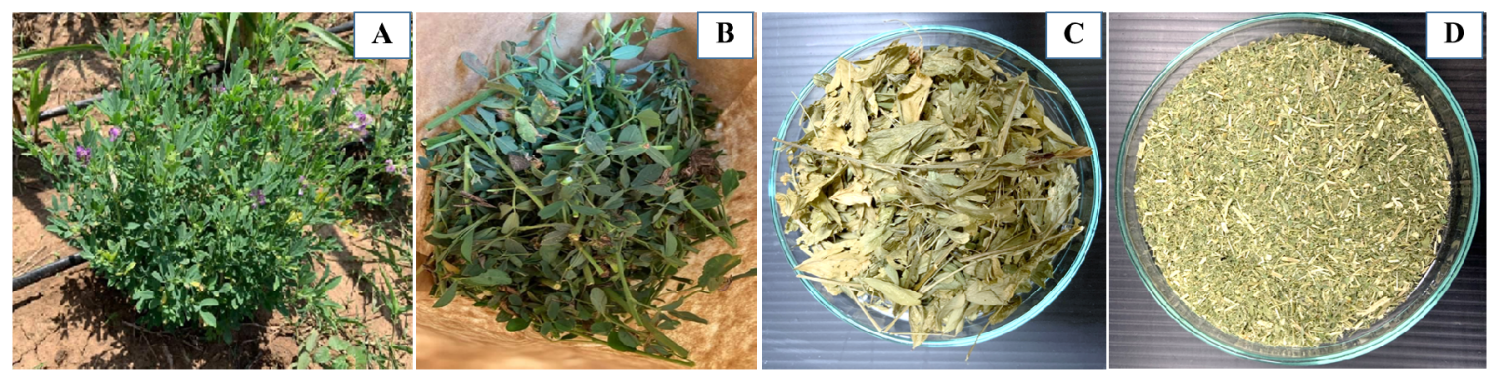

$\mathbf{A}=$ Alfalfa plants grown in experimental farm of the Agricultural Research Facility of the Faculty of Agricultural Technology, Burapha University, Sa Kaco campus, Sa Kaco, Thailand.

$\mathbf{B}=$ The fresh alfalfa plants samples were collected at harvesting intervals of 75 days with the third cut cycle (225 days).

$\mathrm{C}=$ Pre-drying (B samples) was dried at $60^{\circ} \mathrm{C}$ for at least $48 \mathrm{~h}$.

$\mathbf{D}=$ Ground samples (C samples) for chemical composition analysis.

Figure 1 Plantation and collection of AP material for chemical composition analysis.

\section{Chemical analysis of alfalfa plants (AP)}

An analysis of the chemical composition of AP samples was conducted to evaluate their nutrional values, including dry matter (DM), OM, CP, ether extract (EE), ash, neutral-detergent fiber (NDF), aciddetergent fiber (ADF), acid-detergent lignin (ADL), and tannin. Regarding the dried AP samples, the process was repeated for DM content with oven drying at $135^{\circ} \mathrm{C}$ for $2 \mathrm{~h}$ to a constant weight following a method based on the Association of Official Analytical Chemists (AOAC) [7] official method recommendations as in the procedure described in 2016 (method 930.15). The CP was analyzed using an in-house method based on the AOAC [7] (Kjeldahl $\mathrm{N} \times 6.25$, method 2001.11). The EE was determined by using petroleum ether (AOAC as in the procedure described in 2010 [8]); method 2003.05). Ash was analyzed following the AOAC [7] (method 942.05). The CF was determined by the standard methods of the AOAC method 962.09 [8]. The NDF, ADF and ADL were determined using the method described by Van Soest et al. [9] adapted for a Fiber Analyzer. Sodium sulfite was used in the neutral detergent solution for NDF analysis. The NDF and ADF are expressed inclusive of residual ash. Determination of tannins (on a DM basis) was carried out by following Burns as described in a procedure in 1971 [10]. The chemical analysis was expressed on the basis of the final DM. The analyses were performed on each sample in 3 replicates.

\section{Chemical analysis of alfalfa pellets (APe)}

The DM was conducted following the standard methods of AOAC [7] (method 930.15), CP (method 2001.11) [7], EE (method 2003.05) [8] respectively, and analyzed according to AOAC [7] for ash (method 942.05), and CF (method 962.09) [8]. The NDF, ADF, and ADL were analyzed following the method of Van Soest et al. [9]. Determination of tannins was carried out by following Burns [10].

\section{Total phenolic content (TPC)}

The extracts prepared from the $5 \mathrm{~g}$ dried samples with $20 \mathrm{~mL}$ of $80 \%$ methanol (v/v) on a shaking incubator set at $37^{\circ} \mathrm{C}$ for $12 \mathrm{~h}$. The extracted sample was filtered through a Whatman No. 1 filter. After mixing supernatant with other previously extracted supernatants of the sample under identical conditions and combined before being decanted into a vial and then stored at $-20^{\circ} \mathrm{C}$ until the TPC, TFC and anitioxidant activity were measured. To determine the contents of TPC Folin-Ciocalteu's reagent was used as previously described by Kubola and Siriamornpun [11] and then compared to the standard of gallic acid. Three hundred microliters of sample extract were mixed with $10 \%$ of the Folin-Ciocalteu reagent and $2 \mathrm{~mL}$ of $7.5 \%$ sodium carbonate $\left(\mathrm{Na}_{2} \mathrm{CO}_{3}\right)$ solution. The absorbancy was measured at 725 $\mathrm{nm}$ using a spectrophotometer, after incubation time at room temperature for $90 \mathrm{~min}$. Gallic acid 
http://wjst.wu.ac.th

equivalents (GAE) at a concentration of 1 to $1,000 \mathrm{mg} / \mathrm{L}$ which was used as a standard for expressed the amount of the TPC of the sample extract. The total quantity of phenolics was expressed in milligrams GAE per gram of DW sample (mg GAE/g DW) based on the gallic acid standard curve.

\section{Total flavonoid content (TFC)}

Dried samples were extracted under the same conditions as for TPC. The TFC was determined using the colorimetric method described by Kubola and Siriamornpun [11]. To determine the total amount of flavonoid compounds, $500 \mu \mathrm{L}$ of the sample extracts were mixed with $2.25 \mathrm{~mL}$ of distilled water. The extracted samples were added with $150 \mu \mathrm{L}$ of $5 \% \mathrm{NaNO}_{2}$ solution. After $6 \mathrm{~min}, 300 \mu \mathrm{L}$ of a $10 \%$ $\mathrm{AlCl}_{3} .6 \mathrm{H}_{2} \mathrm{O}$ solution was added and allowed to stand for another $5 \mathrm{~min}$ before $1.0 \mathrm{~mL}$ of $1 \mathrm{M} \mathrm{NaOH}$ was added. The mixture was mixed well using a vortex, then the absorbance was taken at $510 \mathrm{~nm}$ using a UVvis spectrophotometer. The results were expressed in $\mathrm{mg}$ rutin equivalents in $1 \mathrm{~g}$ of the dried sample (mg $\mathrm{RE} / \mathrm{g})$.

Phenolic acids (PC) and flavonoid compounds (FC)

The PC and FC were carried out according to the method described previously by Kubola et al. [12] with some modifications. Each sample $(5 \mathrm{~g})$ was mixed with $50 \mathrm{~mL}$ of methanol/HCL $(100: 1, \mathrm{v} / \mathrm{v})$ in a shaking incubator for $12 \mathrm{~h}$ at $37{ }^{\circ} \mathrm{C}$. The extract was then centrifuged at $8,000 \mathrm{rpm} / \mathrm{min}$, and the supernatant was filtered through the Whatman No. 4 filter. The concentration of the residue was increased by separating solvents using a rotary evaporator at $40{ }^{\circ} \mathrm{C}$ until the volume was less than $5 \mathrm{~mL}$. The residue was re-dissolved in $5 \mathrm{~mL}$ of methanol/water $(80: 20, \mathrm{v} / \mathrm{v})$ and filtered through a $0.45 \mu \mathrm{m}$ filter before injection $20 \mu \mathrm{L}$ ) into a high-performance liquid chromatography (HPLC) aperture. Samples were analyzed in triplicate. HPLC-DAD system for analysis of PC and FC: the reverse phase RP-HPLC system for analysis of phenolic compounds HPLC analysis was performed using Shimadzu LC-20AC pumps, an SPD-M20A diode array detector and chromatographic separations were performed on a LUNA C-18 column $\left(4.6 \times 250 \mathrm{~mm}^{2}\right.$ i.d., $\left.5 \mu \mathrm{m}\right)$. The mobile phase was under gradient elution conditions. The mobile phase consisted of purified water with $1 \%$ acetic acid (solvent A) and the other mobile phase was acetonitrile ( solvent B). Gradient elution was performed as follows: from 0 to 40 min, linear gradient from 30 to $70 \%$ solvent B; from 40 to $45 \mathrm{~min}, 20$ to $80 \%$ solvent B; from 45 to 55 min, linear gradient from 15 to $85 \%$ solvent B; from 55 to 57 min, linear gradient from 10 to $90 \%$ solvent $\mathrm{B}$. The operating conditions of column temperature were $38^{\circ} \mathrm{C}$, injection volume, $20 \mu \mathrm{L}$, and photo diode array detection at 280, 320 (phenolic), and $370 \mathrm{~nm}$ (flavonoid). The PC and FC in the samples were identified by comparing their relative retention times and the substance compared to the standard substances of PC and FC.

\section{Isoflavone content}

The determination of isoflavone content was conducted as previously described by Akitha et al. [13]. Samples of $5 \mathrm{~g}$ of dried finely ground samples were added to $50 \mathrm{~mL}$ of $80 \%$ methanol for extraction for $12 \mathrm{~h}$ at $35^{\circ} \mathrm{C}$ in a rotary shaker set at $150 \mathrm{rpm} / \mathrm{min}$. After the extraction time was over, the solution was filtered through Whatman No. 1 filter paper. The filtrate was kept at $-20{ }^{\circ} \mathrm{C}$ until analysis and then subsequently analyzed by HPLC to obtain the isoflavone from the samples. Analysis of the isoflavone content was performed using HPLC; Shimadzu LC-20AC pumps, an SPD-M20A diode array detector, and a LUNA C-18 column $\left(4.6 \times 250 \mathrm{~mm}^{2}\right.$ i.d., $\left.5 \mu \mathrm{m}\right)$. Before analysis, the sample extract was filtered through a $0.45 \mu \mathrm{m}$ filter and transferred into HPLC vials. The mobile phase consisted of 3 phases as follows: phase A used a purified DI water (ratio $50 \%$ ), phase B used acetonitrile (ratio $10 \%$ ) (solvent B) and phase D used $1 \%$ acetic acid in DI water (v/v) (ratio $40 \%$ ) with a setting under isocratic conditions. A $20 \mu \mathrm{L}$ of the sample extract was injected at a flow rate of $1 \mathrm{~mL} / \mathrm{min}$ from 0 to $30 \mathrm{~min}$. The column was measured at a wavelength of $277 \mathrm{~nm}$, compared to the duration of the standard substance (genestein). 
http://wjst.wu.ac.th

\section{Amino acids by LCMS/MS}

Sample extraction for the analysis of AA analysis was conducted following Thiele et al. [14]. A 0.5 $\mathrm{mL}$ aliquot of $0.5 \mathrm{M}$ aqueous HCL-ethanol $(1: 1, \mathrm{v} / \mathrm{v})$ as solvent was added to $100 \mathrm{~g}$ of dried and ground material, then the extract was centrifuged at $12,000 \times \mathrm{g}$ for $15 \mathrm{~min}$ at $4{ }^{\circ} \mathrm{C}$, and the supernatant was separated directly and used for analysis. Samples were analyzed using the AA method as performed by Nimbalkar et al. [15] with some modifications. The LC-MS-MS analysis system consists of the Shimadzu LCMS-8030 triple-quadrupole mass spectrometer (Shimadzu, Kyoto, Japan) operating system, ESI mode (electrospray ionization), and HPLC system (Shimadzu LC-20AC series, Kyoto, Japan) consisting of a vacuum degasser with a binary pump, a thermostated column oven, and an autosampler. Gradient eluent was operated by the column couple InertSustain ${ }^{\circledR}$ C-1 $8 \quad\left(2.1 \times 150 \mathrm{~mm}^{2}, 3 \mu \mathrm{m}\right)$ with guard column InertSustain ${ }^{\circledR} \mathrm{C}-18\left(2.1 \times 10 \mathrm{~mm}^{2}, 3 \mu \mathrm{m}\right)$. The mobile phase A consisted of purified water with formic 0.1 $\%$ and methanol/water $(50: 50, \mathrm{v} / \mathrm{v}$ ) mixed with formic $0.1 \%$ (phase B) at a flow rate of $0.2 \mathrm{~mL} / \mathrm{min}$. Gradient elution was performed for mobile phase B as follows: from 0 to $1 \mathrm{~min} / 2 \%$ (v/v), from 1 to 10 $\mathrm{min} / 2-80 \%(\mathrm{v} / \mathrm{v})$; from 10 to $12 \mathrm{~min} / 80 \%$ (v/v), from 12 to $13 \mathrm{~min} / 80-2 \%(\mathrm{v} / \mathrm{v})$, and from 12 to 15 $\min / 2 \%(\mathrm{v} / \mathrm{v})$. The initial condition was as follows: $20 \%$ eluent $\mathrm{B}$, with the column set at $38{ }^{\circ} \mathrm{C}$. Injections were carried out with an autosampler operating at $4{ }^{\circ} \mathrm{C}$ and the autosampler was washed before and after the injection of the samples using methanol as a nebulizing gas cleaner and dried gas as nitrogen at a flow rate of 3.0 and $15.0 \mathrm{~L} / \mathrm{min}$ with the ion source voltage of $4.5 \mathrm{kV}$. The DL temperature was 250 ${ }^{\circ} \mathrm{C}$ and the heat block temperature was $400{ }^{\circ} \mathrm{C}$. The multiple reaction monitoring (MRM) conditions for AA on LCMS/MS, where argon was generated from gas collision (CID) at $230 \mathrm{kPa}$, with the detector voltage, were set at $1.72 \mathrm{kV}$.

\section{Antioxidant activity \\ DPPH radical scavenging assay}

The scavenging activity of the extracts was estimated by using 2, 2-diphenyl-1-picrylhydrazyl (DPPH) free radicals and a method adapted from Wanyo et al. [16]. Aqueous extract $(0.1 \mathrm{~mL})$ was added to $3 \mathrm{~mL}$ of a solution of $0.001 \mathrm{M}$ DPPH in methanol. The mixture was shaken vigorously and left to stand for $30 \mathrm{~min}$ in the dark at room temperature. The absorbance was measured spectrophotometrically at 517 $\mathrm{nm}$. The activity was calculated according to an equation of percentage of free radical scavenging effect $=$ $\left.\left[\left(\mathrm{A}_{\mathrm{DPPH}}-\mathrm{A}_{\mathrm{SAMPLE}}\right) / \mathrm{A}_{\mathrm{DPPH}}\right)\right] \times 100\left(\mathrm{~A}_{\mathrm{DPPH}}\right.$ denotes absorbance without extract whilst $\mathrm{A}_{\mathrm{SAMPLE}}$ denotes absorbance with extract). A standard of Trolox was utilized as a reference substance from the beginning 1 to $500 \mu \mathrm{g} / \mathrm{mL}$. The results were expressed as the Trolox equivalent antioxidant capacity in mg Trolox eq./g dried sample and calculated from a calibration curve of Trolox.

\section{Ferric reducing antioxidant power (FRAP) assay}

Antioxidant activity of the sample extracts were determined by the FRAP assay adapted from Wanyo et al. [16] and Kubola et al. [12]. The FRAP reagent was prepared by mixing as the following: $100 \mathrm{~mL}$ of acetate buffer $(300 \mathrm{mM}, \mathrm{pH} 3.6), 10 \mathrm{~mL}$ TPTZ solution (10 mM TPTZ in $40 \mathrm{mM} \mathrm{HCl}), 10 \mathrm{~mL}$ $\mathrm{FeCl}_{3} \cdot 6 \mathrm{H}_{2} \mathrm{O}(20 \mathrm{nM})$ in a ratio of $10: 1: 1$ and $12 \mathrm{~mL}$ distilled water, and the mixtures were incubated at 37 ${ }^{\circ} \mathrm{C}$. To perform the FRAP assay, $60 \mu \mathrm{L}$ sample and $180 \mu \mathrm{L}$ Milli-Q water were mixed with $1.8 \mathrm{~mL}$ of FRAP reagent, standard or blank tubes were then added to the test tubes, and incubated at $37{ }^{\circ} \mathrm{C}$ for 4 min, absorbance was read at $593 \mathrm{~nm}$, using the FRAP working solution as a blank. FRAP values of the sample based on the FRAP assay were reported as $\mathrm{mgFe}^{2+}$ per gram of dried sample $\left(\mathrm{mgFe} \mathrm{g}^{2+} / \mathrm{g}\right)$.

\section{Statistical analysis}

All data of the analysis of the chemical composition, bioactive compounds, AA, and antioxidant activities of AP and APe were evaluated for variances and differences by using the F-test and the mean differences were analyzed using a t-test with $\mathrm{R}$ statistics [17]. The results are presented as means \pm standard deviation (SD) of triplicate observations with a probability level of 0.05 significance. Correlation coefficients among TPC, TFC, DPPH free radical scavenging activities, and FRAP assay 
http://wjst.wu.ac.th

were calculated by using the Analysis ToolPak in the Microsoft Excel Program to determine the relationships among traits. Probability was considered to be significant at $<0.05$.

\section{Results and discussion}

The DM, OM, CP, EE, ash, CF, NDF, ADF, ADL and tannin values of the AP and APe are presented in Table 1. The moisture of the APe samples was lower than those of AP due to the practical effects of dehydration in pellet forming. Dehydration requires pre-wilting and chopping in the field, transportation to the plant, and drum-drying down to $10 \%$ moisture [18]. These results show that the ash of APe was remarkably higher than AP, and the results of this study also conform to the previous data which reported the AP which had lower ash than APe [2]. The AP at harvesting intervals of 75 days with the third cut cycle was lower in CP than APe. In general, alfalfa grown in the field in temperate region is harvested during the flowering stage of each cutting, which has a high yield and quality [19]. However, planting alfalfa in Thailand will bloom once a year in winter, therefore the alfalfa harvesting of this experiment depended on the number of days. Whereas, the mechanical grinding and pelleting processinduced some nutrient minor changes to the forage [20]. It is well known that NDF is a component of the cell wall that contains all of the fibers: hemicellulose, cellulose, lignin, cutin, silica, keratin, which are beneficial to ruminants since the rumen of ruminants contains microorganisms that can digest cellulose and hemicellulose. Both AP and APe were similar in having a high content of fiber, especially the proportion of hemicellulose, ruminants fed diets contain the with right proportions of fiber had possibly greater utilization. Lignin is an inhibitor of digestion of hemicellulose that may have reduced digestibility. Tannins were found among all samples of AP and APe, ranging from 1.80 to $3.08 \%$. Tannins are phenolic secondary metabolites of their astringent plants which are also known as phytochemicals [21]. The use of tannin concentrations of not more than $5 \%$ in ruminant feed will affect feed intake and may be toxic to animals [22]. Tannins can increase the production of microbial proteins that can by-pass protein through the small intestine in ruminants [23], so tannins are mechanically reduced and degraded by microbes in the rumen. A larger amount of protein passes through the abomasum, which increases the absorption of essential AA in the small intestine and is also absorbed by the animal itself to increase its growth rate [24]. 
http://wjst.wu.ac.th

Table 1 Comparison of chemical composition (\%) of the AP and APe with the 3 values obtained for each set with SD.

\begin{tabular}{|c|c|c|c|c|c|}
\hline Item & DM & OM & $\mathbf{C P}$ & $\mathbf{E E}$ & Ash \\
\hline AP & $93.44 \pm 1.005$ & $85.33 \pm 0.963$ & $16.21 \pm 0.067$ & $1.42 \pm 0.101$ & $8.12 \pm 0.100$ \\
\hline $\mathrm{APe}$ & $96.53 \pm 0.594$ & $80.65 \pm 0.702$ & $18.17 \pm 0.506$ & $1.69 \pm 0.102$ & $15.89 \pm 1.277$ \\
\hline$P$ value- $T$ & $\begin{array}{c}0.01 \\
* *\end{array}$ & $\begin{array}{c}0.01 \\
* *\end{array}$ & $\begin{array}{c}0.05 \\
*\end{array}$ & $\begin{array}{c}0.05 \\
*\end{array}$ & $\begin{array}{c}0.001 \\
* * *\end{array}$ \\
\hline Item & $\mathrm{CF}$ & NDF & ADF & ADL & Tannins \\
\hline $\mathrm{AP}$ & $26.63 \pm 0.332$ & $45.81 \pm 0.074$ & $38.47 \pm 0.702$ & $9.89 \pm 0.076$ & $1.80 \pm 0.031$ \\
\hline $\mathrm{APe}$ & $25.03 \pm 1.004$ & $39.28 \pm 0.326$ & $31.11 \pm 0.140$ & $6.07 \pm 0.663$ & $3.08 \pm 0.060$ \\
\hline$P$ value-T & $\begin{array}{c}0.05 \\
*\end{array}$ & $\begin{array}{c}0.001 \\
* * *\end{array}$ & $\begin{array}{c}0.01 \\
* *\end{array}$ & $\begin{array}{c}0.01 \\
* *\end{array}$ & $\begin{array}{c}0.01 \\
* *\end{array}$ \\
\hline
\end{tabular}

$\mathrm{AP}=$ Alfalfa plants (The representation of the $1^{\text {st }}$ to $3^{\text {rd }}$ replicated alfalfa plants at harvesting intervals of 75 days with the $3^{\text {rd }}$ cut cycle results are an average of the 3 values obtained for each set with a standard deviation), APe $=$ Alfalfa pellets in January, March and June 2019, DM $=$ Dry matter, $\mathrm{OM}=$ Organic matter, $\mathrm{CP}=$ Crude protein, $\mathrm{EE}=\mathrm{Ether}$ extract, $\mathrm{CF}=$ Crude fiber, $\mathrm{NDF}=$ Neutral detergent fiber, $\mathrm{ADF}=$ Acid detergent fiber, $\mathrm{ADL}=$ Acid detergent lignin, * Significance at $\mathrm{p}<0.05, * *$ Significance at $\mathrm{p}<0.01, * * *$ Significance at $\mathrm{p}<0.001$

Table 2 Quatification of TPC, TFC, PC, FC, isoflavone content and AA in AP and APe.

\begin{tabular}{|c|c|c|c|}
\hline Items & $\mathbf{A P}$ & APe & p-value (T) \\
\hline TPC (mgGAE/g) & $5.24 \pm 0.26$ & $2.42 \pm 0.02$ & $* *$ \\
\hline TFC (mgRE/g) & $6.65 \pm 0.10$ & $2.25 \pm 0.10$ & $* * *$ \\
\hline DPPH (mg Trolox eq./g) & $28.83 \pm 2.75$ & $1.58 \pm 0.09$ & $* *$ \\
\hline FRAP $\left(\mathrm{mgFe}^{2+} / \mathrm{g}\right)$ & $2.09 \pm 0.11$ & $3.25 \pm 0.08$ & $* * *$ \\
\hline \multicolumn{4}{|l|}{ Phenolic compounds $(\mu \mathrm{g} / \mathrm{g} D W)$} \\
\hline Gallic acid (GA) & $3.14 \pm 0.37$ & $215.30 \pm 3.35$ & $* * *$ \\
\hline Protocatechuic acid (PCCA) & nd & $78.55 \pm 1.47$ & \\
\hline p-hydroxybenzoic acid (p-HO) & $74.39 \pm 1.67$ & $131.03 \pm 3.07$ & $* * *$ \\
\hline Chlorogenic acid (ChA) & nd & $56.29 \pm 3.57$ & \\
\hline Vanilic acid (VA) & $4.24 \pm 0.08$ & $78.79 \pm 2.81$ & $* * *$ \\
\hline Caffeic acid (CFA) & $11.48 \pm 0.01$ & $143.58 \pm 4.77$ & $* * *$ \\
\hline Syrigic acid (SyA) & $2.79 \pm 0.03$ & $17.22 \pm 0.84$ & $* * *$ \\
\hline p-Coumaric acid (p-CA) & $64.82 \pm 0.65$ & $53.91 \pm 3.09$ & $*$ \\
\hline Ferulic acid (FA) & $22.25 \pm 2.53$ & $142.22 \pm 5.54$ & $* * *$ \\
\hline Sinapic acid (SNA) & $24.36 \pm 1.68$ & $18.72 \pm 1.99$ & $* *$ \\
\hline Total & $207.47 \pm 27.26$ & $935.61 \pm 63.18$ & \\
\hline \multicolumn{4}{|l|}{ Flavonoid compounds $(\mu \mathrm{g} / \mathrm{g} D W)$} \\
\hline Catechin & $679.49 \pm 15.68$ & $430.59 \pm 6.69$ & \\
\hline Rutin & $264.57 \pm 4.55$ & $42.28 \pm 3.41$ & $* * *$ \\
\hline Myricetin & $3910.40 \pm 425.57$ & $1378.73 \pm 86.84$ & $* *$ \\
\hline Luteolin & $453.01 \pm 14.10$ & $19.04 \pm 0.51$ & \\
\hline Quercetin & nd & $181.28 \pm 10.40$ & \\
\hline Apigenin & $280.76 \pm 3.95$ & $2278.27 \pm 90.20$ & $* * *$ \\
\hline Total & $5588 \pm 1645.15$ & $4330.61 \pm 915.07$ & \\
\hline
\end{tabular}

\section{Isoflavone $(\mu \mathrm{g} / \mathrm{g} \mathrm{DW})$}




\begin{tabular}{lccc}
\hline Items & AP & APe & p-value (T) \\
\hline Genistein & $104.37 \pm 3.78$ & $25.95 \pm 0.00$ & $* * *$ \\
Daizein & $130.91 \pm 5.15$ & $55.12 \pm 0.01$ & $* * *$ \\
\hline Amino acids $(\boldsymbol{\mu g} / \mathbf{g}$ DW) & & & \\
\hline Histidine & - & $33.14 \pm 3.87$ & \\
Isoleucine & $0.08 \pm 0.00$ & $3177.30 \pm 21.50$ & $* * *$ \\
Leucine & $0.20 \pm 0.00$ & $3908.11 \pm 38.10$ & $* * *$ \\
Lysine & $0.01 \pm 0.00$ & $135.54 \pm 1.76$ & $* * *$ \\
Methionine & $0.03 \pm 0.00$ & $11.74 \pm 0.94$ & $* *$ \\
Phenylalanine & $4.30 \pm 0.08$ & $2544.94 \pm 13.03$ & $* * *$ \\
Threonine & $0.21 \pm 0.01$ & $419.34 \pm 14.16$ & $* * *$ \\
Tryptophan & $1.42 \pm 0.02$ & $865.83 \pm 7.33$ & $* * *$ \\
Valine & $3.08 \pm 0.15$ & $5451.31 \pm 46.43$ & $* * *$ \\
Arginine & - & $142.22 \pm 14.05$ & \\
TEA & $9.33 \pm 0.24$ & $16689.47 \pm 155.66$ & $* * *$ \\
TAA & $9.33 \pm 0.24$ & $16831.69 \pm 169.71$ & $* * *$ \\
\hline
\end{tabular}

Values are expressed as mean \pm SD of triplicate measurements.

TPC $=$ Total phenolic content, $($ TPC was determined in comparison with standard gallic acid and the results expressed in terms of $\mathrm{mg} \mathrm{GAE} / \mathrm{g}$ ), TFC $=$ Total flavonoid content (TFC was determined by comparison with standard rutin and the results expressed in terms of $\mathrm{mg} \mathrm{RE} / \mathrm{g}$ ), $\mathrm{DPPH}=$ Radical scavenging assay, FRAP $=$ Ferric reducing antioxidant power assay, $\mathrm{AP}=$ Alfalfa plants, $\mathrm{APe}=$ Alfalfa pellets, $\mathrm{TEA}=$ Total essential amino acid, TAA $=$ Total amino acid, $n d=$ Not detected, ${ }^{*}$ Significance at $\mathrm{p}<0.05,{ }^{* *}$ Significance at $\mathrm{p}<0.01,{ }^{* * *}$ Significance at $\mathrm{p}<0.001$

The average values of TPC and TFC of APe in this study were $2.42 \mathrm{mg} \mathrm{GAE} / \mathrm{g}$, and $2.25 \mathrm{mg} \mathrm{RE} / \mathrm{g}$, respectively, less than AP (5.24 mg GAE/g, and $6.65 \mathrm{mg} \mathrm{RE} / \mathrm{g}$, respectively (Table 2)). Kagan et al. [25] reported that the TPC of alfalfa in 27 species using Folin-Ciocalteu colorimetric assay was $15.8 \pm 1.4 \mathrm{mg} / \mathrm{g}$ DW. Different standard phenolic values yielded a response factor in different regions when analyzed with a colorimetric assay. Several studies have shown that the TPC in the legumes varies depending on the legume types or the growth period, such as ungerminated legumes, germinated legumes, or beans that have been fermented or processed through heat (dry) following Dajanta and Rongkom [26]. 


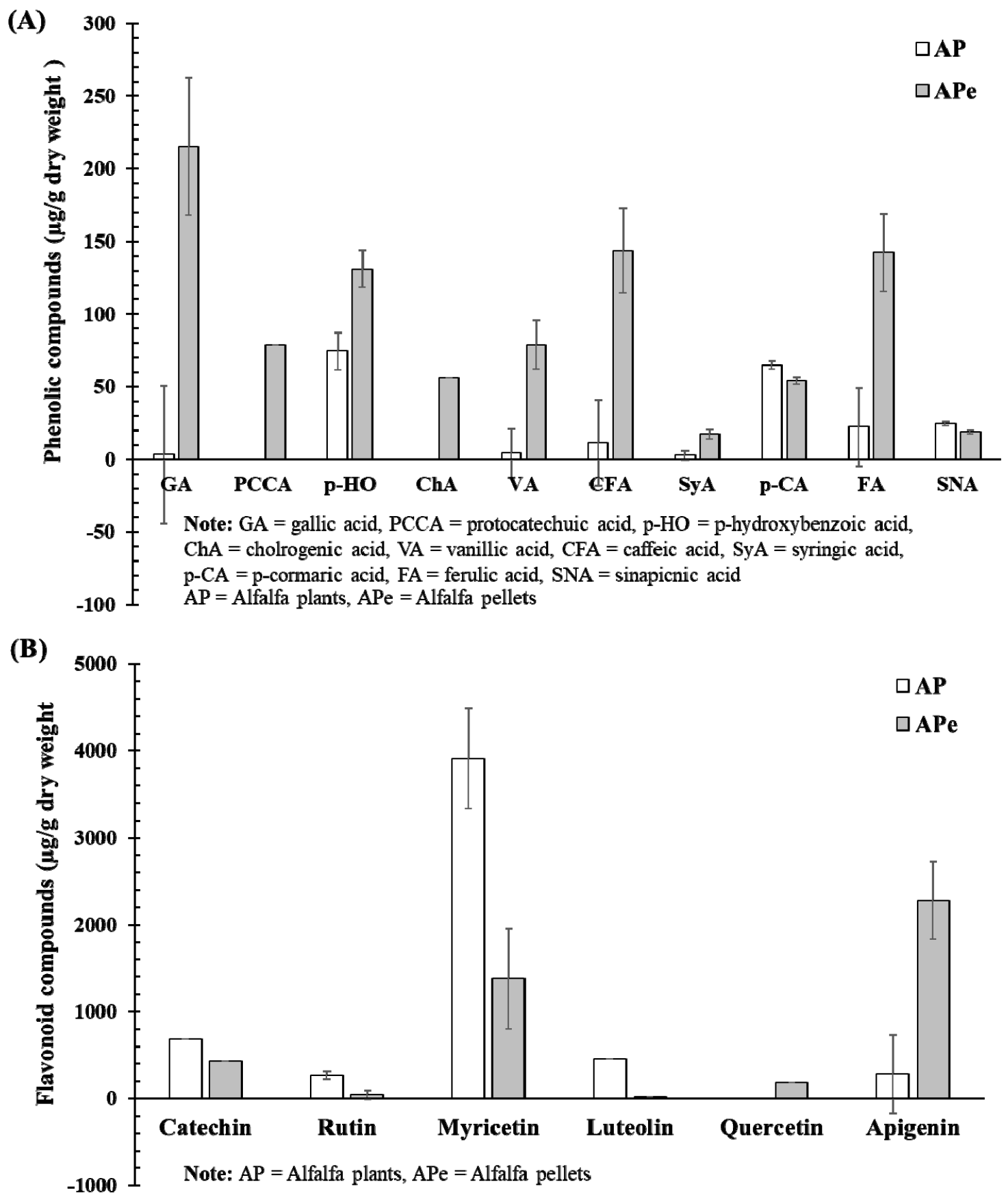

Figure 2 Contents of phenolic (A) and flavonoid (B) in AP and APe.

The detection and quantification of phenolic acids, namely, hydroxybenzoic acids ( gallic acid, protocatechuic acid, and p-hydroxybenzoic acid) and hydroxycinnamic acids (chlorogenic acid, caffeic acid, p-coumaric acid, ferulic acid, and sinapic acid), syrigic acid, and vanilic acid. The PC in APe was the highest in gallic acid, and lowest in syrigic acid (Table 2). On the other hand, gallic acid and syrigic 
http://wjst.wu.ac.th

acid were the lowest, and protocatechuic acid and chlorogenic acid were absent in the AP samples. Wang et al. [27] studied the phenolic acid contents and allelopathic potential of 10-cultivars of alfalfa and their bioactivity. They found that the highest quantities of exuded allelochemicals phenolic acids included: phydroxybenzoic acid, caffeic acid, chlorogenic acid, p-coumaric acid, ferulic acid, cinnamic acid and total phenolic acids of aqueous extract from roots of alfalfa, including of Hunterriver, Longdong, Aohan, Surprise, Pioneer, Victoria, Post mark, WL-525HQ, WL903 and WL-343HQ cultivars. The aerial parts of 10-cultivars of alfalfa $(\mu \mathrm{g} / \mathrm{g} \mathrm{FW})$ contained a different level of phenolic acids in ranges: phydroxybenzoic acid, caffeic acid, chlorogenic acid, coumaric acid, ferulic acid, cinnamic acid, and total phenolic acids, while the p-hydroxybenzoic acid from Surprise, Victoria and WL-903 cultivars were at $39.18,36.85$ and $35.97 \mu \mathrm{g} / \mathrm{g}$, respectively, which was higher than that of other alfalfa species. Wang et al. [27] clearly maintain that the phenolic acids of the aqueous extract from roots also vary according to the legume species. There are differences in the allelochemicals phenolic acids extracted from the various tissues of alfalfa [28]. According to studies in the roots and aerial parts of alfalfa [27], non-detectable gallic acid, protocatechuic acid, vanilic acid, syrigic acid and sinapic acid can occur. Different parts of the alfalfa sample lead to different phenolic types and levels. Therefore, identify the types and quantities of $\mathrm{PC}$ in the different types or forms of cultivars and cutting harvest of alfalfa samples is difficult because they are very diverse and varied in volume.

The flavonoids in AP and APe are presented in Table 2 and Figure 2. Six flavonoids were identified in APe: catechin, rutin, myricetin, luteolin, quercetin and apigenin, while myricetinin was abundant in AP. Interestingly, APe showed the highest apigenin content $(2278.27 \pm 90.20 \mu \mathrm{g} / \mathrm{g})$, followed by myricetin $(1378.73 \pm 86.84 \mu \mathrm{g} / \mathrm{g})$, catechin, quercetin, rutin and luteolin $(430.59 \pm 6.69,181.28 \pm 10.40$, $42.28 \pm 3.41$ and $19.04 \pm 0.51 \mu \mathrm{g} / \mathrm{g})$, respectively. Some parts of apigenin and luteolin structures have been detected in alfalfa legumes, and glucuronic acid was found for the $1^{\text {st }}$ time in aerial parts of alfalfa on the experimental farm of the Institute of Soil Science and Plant Cultivation in Pulawy [3]. It is known that apigenin and luteolin belong to this subclass of flavonoids, also, it restrained some cancer cell lines growth and is anti-inflammatory with free radical scavenging properties [29]. Flavonoids in the chromatography are classified as glycosides of apigenin, luteolin, and tricin [3]. Flavonoids are structuredependent on physiological activity and they also have pharmacological benefits. Thus, these characteristics of the phytochemical components of alfalfa offer potential sources for important healthbased food and/or animal feed compounds and can also help our understanding of their nutritional value.

Data on the isoflavones in the aglycone group (genistein and daidzein) reveal a higher level of daidzein than genistein both AP and APe. (Table 2 and Figure 3). Isoflavone is classified as a group of phytoestrogen and has the effect of preventing other estrogen-related conditions caused by aging, especially among women entering menopause, such as hormone-dependent cancers, cardiovascular diseases, menopausal symptoms, post-menopausal osteoporosis, and neuro-protective effects [30] . Phytoestrogen derived from soybean have been widely researched as an alternative natural functional food supplement to replace estrogen hormones in women entering menopause who are affected when receiving synthetic estrogen hormones over the long-term. Phytoestrogens are plant substances that have estrogenic activity. They signify diverse groups of naturally occurring chemicals with similarities to 17- $\beta$ oestradiol (E2) which is the hormone estrogen [31] found in the human body. Many groups of this plant's substances can be identified, such as isoflavones, chromenes, coumestans, and lignans. However, phytoestrogens can be found in types of legumes, cereals, and grasses, all of which are plants used in the farming of ruminants. Therefore, farmers who manage to feed cows with legumes, soybeans, soy products, and green beans, etc., should be able to produce high phytoestrogen milk that will have benefits which include antioxidant effects, prevention of symptoms of premature aging, and reduction of the risks of NCDs diseases. These conditions include standing issues related to the menopause [32]. Previous research studies showed that dairy cattle fed with legume silage consisting of white clover (Trifolium repens $\mathrm{L}$ ), lucerne (Medicago sativa L.), and red clover (Trifolium pratense L.) detected the concentration of equol present in milk [33, 34]. Steinshamn et al. [33] stated that daidzein, a derivative of the isoflavone group metabolized during digestion and absorbent of equol by intestinal bacteria is superior to other isoflavones, such as the daidzein-based substrate, in its antioxidative activity. According to Sunita and Pattanayak [30], phytoestrogens are plant-derived hormone-like diphenolic compounds of dietary origin 
http://wjst.wu.ac.th

which contain small amounts of estrogen. Steinshamn et al. [33] suggested that dairy cattle fed with red clover-based diets may produce high concentrations of equol due to its health benefits. In this present study, a higher level of daidzein was found in AP than APe at 130.91 and $55.12 \mu \mathrm{g} / \mathrm{g}$ DW, respectively (Table 2). Consequently, when supplemented with a high level of daidzein from legumes plant in the dairy cattle diet, the results suggested a possibly greater increment in the concentration of equol observed in raw cow's milk. Furthermore, the findings of Kwak et al. [34] suggest that higher level of both phenolic and isoflavone compounds in Chungkukjang (a fermented soybean paste used in Korea) are positively correlated with higher antioxidant activity.

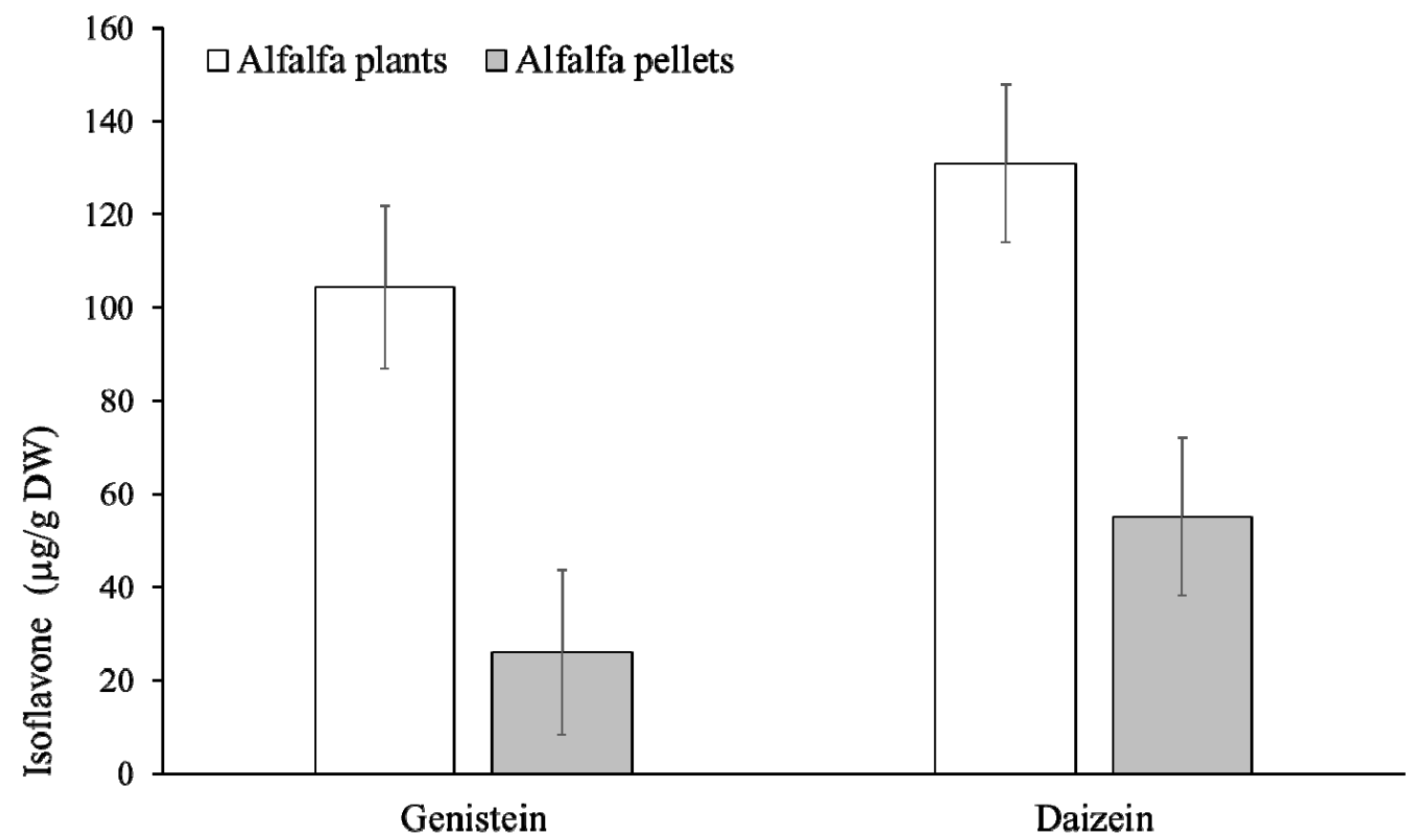

Figure 3 Isoflavone in AP and APe.

The AA of APe revealed the highest amount of valine, followed by leucine, isoleucine, phenylaline, tryptophan, threonine, arginine, lysine, histidine, and methionine (Table 3 and Figure 4), respectively. Normally, protein in a diet provides AA since, after digestion, proteins are converted into AA and absorbed into the small intestine and the bloodstream for use by the animal body, and some are excreted by the kidneys in urine to form urea. The AA of AP were highly varied from APe and they were considerably lower than from APe, which may imply that there exists protein lower than APe. This would be consistent with the results of the analysis of the nutritional value of protein content in Table $\mathbf{1}$. The AA of soybean has a more balanced profile than other types of beans for humans and livestock [11]. Eight types of AA are considered essential AA (isoleucine, leucine, lysine, methionine, phenylalanine, threonine, tryptophan, valine) which must be obtained from various diets and supplements due to the inability of humans and animals to synthesize AA from the beginning (de novo). Histidine is the $9^{\text {th }}$ essential AA required for children and infants. Arginine is one of the non-essential AA which is called a conditionally essential AA that can be generated by the body. Peptides and free AA are known to be a source of antioxidants in addition to phenolic content in traditional Chinese salt-fermented soybean [35]. Peptides that are isolated from essential AA or peptides that have beneficial biological activity are 
http://wjst.wu.ac.th

alternatives to nutrients for humans as peptides are easier to absorb and less harmful to health than synthetic drugs or other substances. Natural antioxidants, such as tocopherols, catechin, PC, and peptides, are safer and less toxic than synthetic antioxidants. However, biologically active peptides consist of 3-20 AA. Many bioactive peptides are used as antioxidants [36], therefore biological activity depends on the sequence of AA that are the components of the peptide.

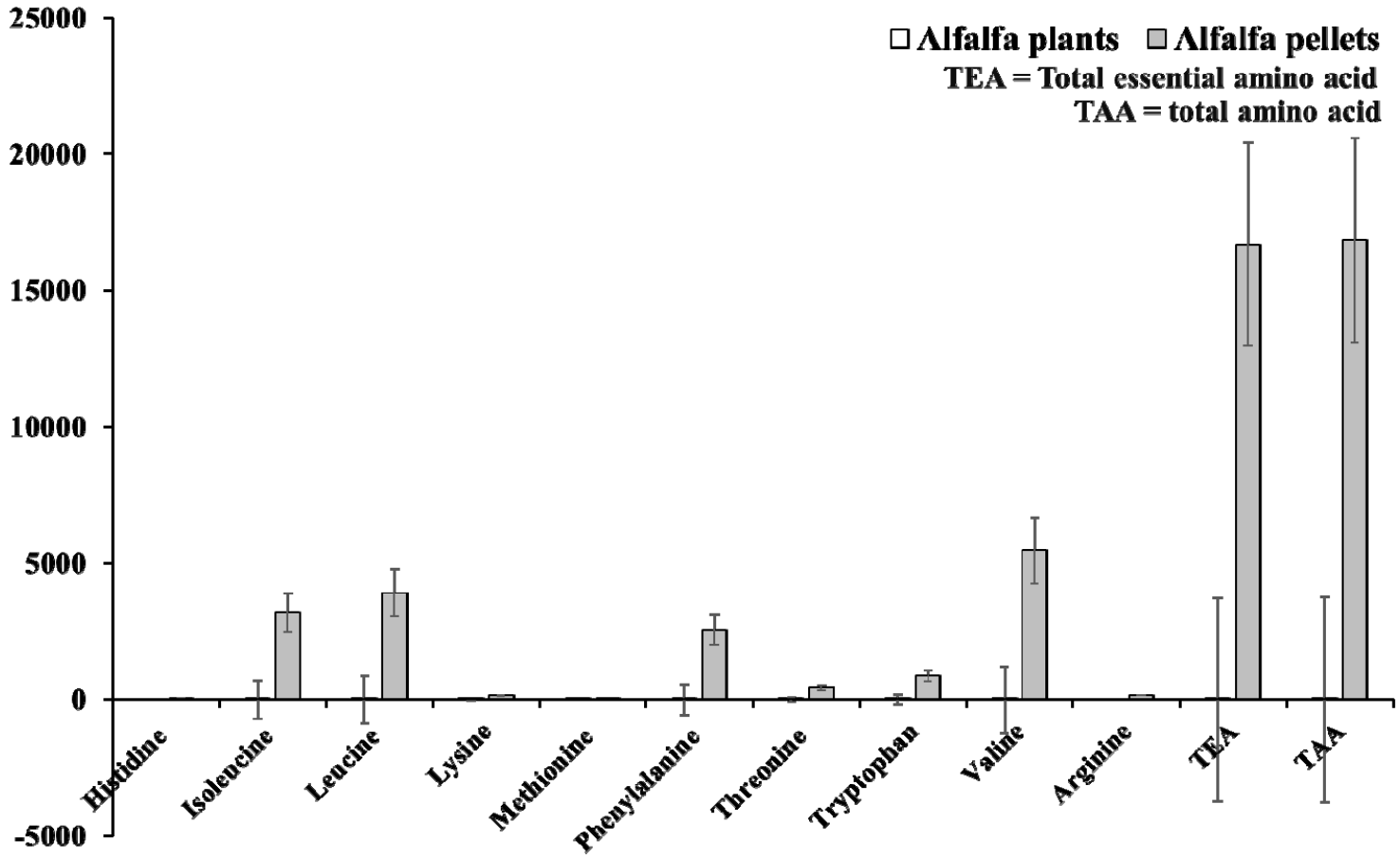

Figure 4 Contents of AA $(\mu \mathrm{g} / \mathrm{g} D W)$ in AP and APe.

Table 2 and Figure 5 show the antioxidative activities of crude extracts of AP and APe samples which were studied with the DPPH free radical scavenging activities and FRAP in AP and APe. The analysis of the antioxidant activities in APe displayed is based on the DHHP radical scavenging assay which accounts for $1.58 \mathrm{mg}$ Trolox eq. $/ \mathrm{g}$, which is significantly lower than AP which ranged from 25.71 to $30.86 \mathrm{mg}$ Trolox eq./g. The FRAP assay of the APe extract was $3.25 \mathrm{mgFe}^{2+} / \mathrm{g}$, whereas AP was 2.09 $\mathrm{mgFe}^{2+} / \mathrm{g}$. Table 3 shows that TFC has significant positive correlation with TPC, DPPH has a positive significant correlation between TPC, TFC, and FRAP has significant negative correlations with TPC, TFC, and DPPH. The antioxidation activities of the sample also correspond to the total phenolic contained in the sample. Our finding suggested that TPC and TFC may be responsible for antioxidant via DHHP. Some research studies demonstrated that PC in vegetables, fruits, or grains show antioxidant activity [34]. The relationship of phenolic together with its ability to be an antioxidant can explain the high amount of TPC which has high antioxidation ability. Furthermore, the antioxidant activity also totally correlates with both the total phenolic and flavonoid content with the fruit of Thuja orientalis growing in Egypt showing the higher values [37]. The results show an increase in the free radical scavenging capacity of the AP or APe extract which can be explained by the presence of higher phenolic contents. The above findings are consistent with those of other researchers and thus support our data which found that TPC, PC and antioxidative activities in DPPH free radical scavenging activities and 
http://wjst.wu.ac.th

FRAP were detected in both AP and APe. However, the significant antioxidant activity of different parts of the plant was also found [38] in Indian Thuja orientalis.

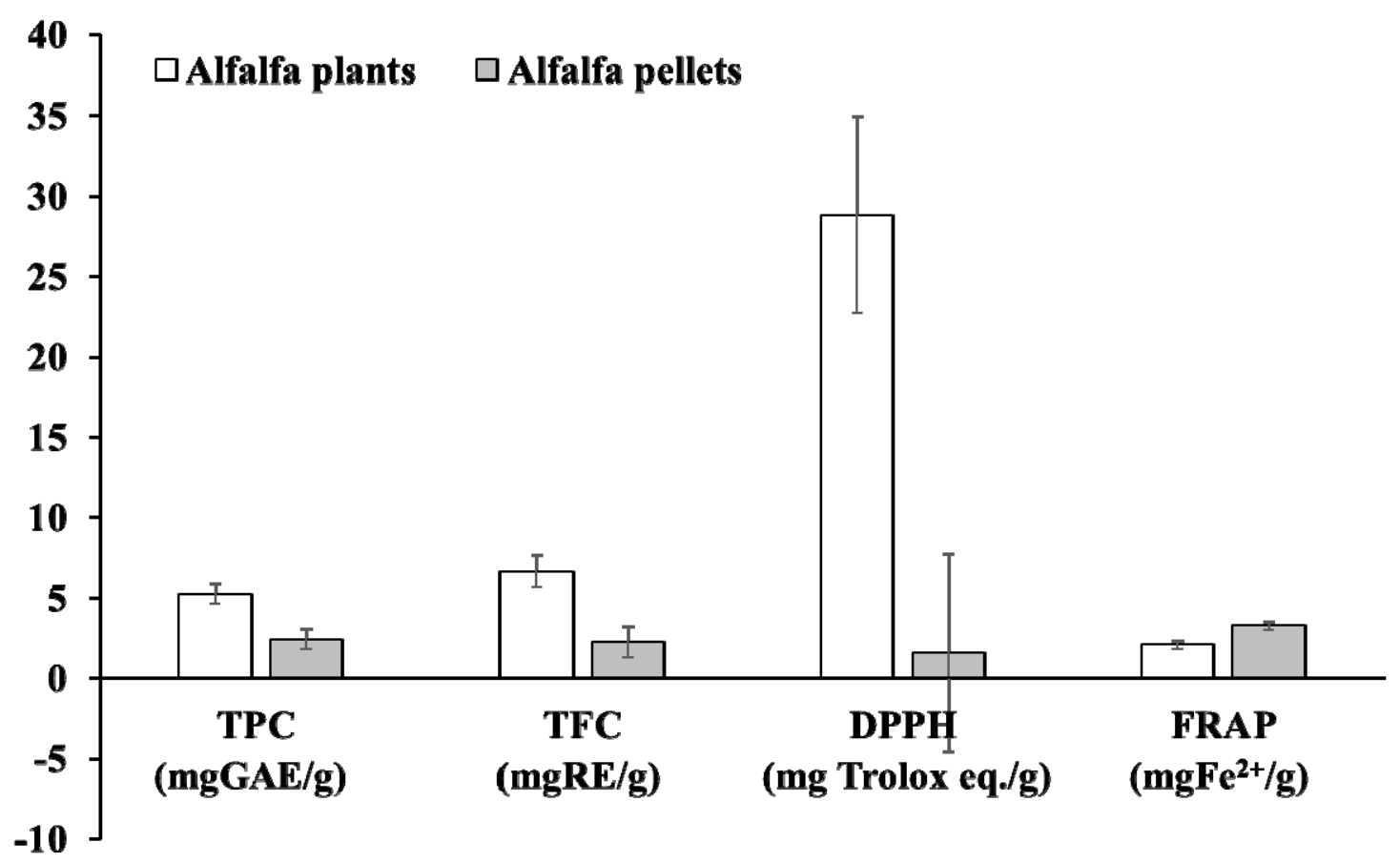

Figure 5 TPC, TFC, and antioxidant activity assessed via DHHP radical scavenging assay including standard (Trolox) and FRAP assay in AP and APe.

Table 3 Correlation coefficient among investigated TPC, TFC, DPPH free radical scavenging activities, FRAP assay of AP, and APe.

\begin{tabular}{lllll}
\hline Item & TPC & TFC & DPPH & FRAP \\
\hline TPC & 1 & & & \\
TFC & $0.99 * * *$ & 1 & 1 & \\
DPPH & $0.98 * * *$ & $0.99 * * *$ & $-0.97 * *$ & 1 \\
FRAP & $-0.99 * * *$ & $-0.99 * * *$ & & \\
\hline
\end{tabular}

DHHP radical scavenging, $\mathrm{FRAP}=$ Ferric reducing antioxidant activity, $\mathrm{AP}=$ Alfalfa plants, $\mathrm{APe}=$ alfalfa pellets, $* *$ Significance at $\mathrm{p}<0.01, * * *$ Significance at $\mathrm{p}<0.001$

Since this research is based on the nutritional values, bioactive compounds, AA, and antioxidant activities of AP and APe for beneficial uses in ruminant feed, this should be acknowledged as a limitation in the conclusions and, thus, the results from this study should be interpreted cautiously. The results of the present studies can be summarized in 3 main points: 1) the nutritional values of proteins components, which are essential for animal growth, 2) the potential of animal production to focus on the production of vital substances in meat or milk as a high-value product to promote health (as also an ingredient added to food for a specific purpose, which may or may not have less nutritional value), 3) the suitability for 
http://wjst.wu.ac.th

practical use of alfalfa on farms. In practice, a long-term use is the best way to obtain high-quality forage over the whole season is to harvest the alfalfa at flowering stages, then dehydrate and stabilize it to preserve its high protein content, vitamins, and overall nutritive value, then APe can be used by Thai farmers. Due to the difficulties of planting alfalfa and its low yield [2], the price of alfalfa seeds is relatively high (approximately $155-160 \mathrm{USD} / \mathrm{kg}$ of alfalfa seed) when purchased commercially. In our previous study, the alfalfa seeds for this study were planted in the forage plot area on Sa Kaeo soil at BUU, with yields per kilogram $(\mathrm{kg})$ per ha of AP lower than other varieties of legumes, such as hamata (approximately 20 - 22 USD/kg of hamata seed) and stylo 'Tha pra' (approximately $6-8 \mathrm{USD} / \mathrm{kg}$ of stylo 'Tha pra' seed) (Data not shown). Therefore, the practical use of AP might be difficult to use as the main roughage source for Thai farmers to raise ruminant animals compared to the use of APe, which is convenient for feeding, and it also provides stable nutritional values and can be easily managed. However, further research on phytochemical compounds utilization in the ruminant fed a diet with AP and/or APe in Thailand is required to evaluate animal productive performance and enhance the value-added profile in raw milk or meat with an increase in the level of bioactive compounds which will offer human consumers greater health benefits.

\section{Conclusions}

Both AP and APe have potential as raw materials in ruminant feed since they contain the necessary nutritional ingredients of protein. The findings obtained from the present study have shown that beneficial bioactive ingredients can have effects on antioxidants via DPPH, particularly with phytoestrogen contents (genistein and daidzein) are a rich source of health benefits which was highly found in AP. However, PC, AA, TEA, TAA in the APe were more abundant than AP.

\section{Acknowledgments}

This work was partial financially supported by the Research Grant of Burapha University through the National Research Council of Thailand (Grant no. 25/2562). The author is grateful to Assistant Professor Dr. Pipat Lounglawan, School of Animal Technology and Innovation, Institute of Agricultural Technology, Suranaree University of Technology, Dr. Quanjai Rupitak in the Faculty of Agricultural Technology, Burapha University Sakaeo Campus, Sa Kaeo, for their technical advice.

\section{References}

[1] DW Nyamai, W Arika, PE Ogola, ENM Njagi and MP Ngugi. Medicinally important phytochemicals: An untapped research avenue. Res. Rev. J. Pharmacogn. Phytochem. 2016; 4, 3449.

[2] S Srisaikham and Q Rupitak. A preliminary study on growth, yield and nutritive value of four varieties of alfalfa and the utilization of alfalfa dehydrated pellets in a total mixed ratio in meat goat diet. Chiang Mai Univ. J. Nat. Sci. 2021; 20, e2021003.

[3] A Stochmal, S Piacente, C Pizza, FD Riccardis, R Leitz and W Oleszek. Alfalfa (Medicago sativa L.) flavonoids. 1. Apigenin and luteolin glycosides from aerial parts. J. Agric. Food. Chem. 2001; 49, 753-8.

[4] TN Barry and WC McNabb. The implications of condensed tannins on the nutritive value of temperate forages fed to ruminants. Br. J. Nutr. 1999; 81, 263-72.

[5] MJ Messina. Legumes and soybeans: Overview of their nutritional profiles and health effects. Am. J. Clin. Nutr. 1999; 70, 439-50.

[6] Q Wang, X Ge, X Tian, Y Zhang, J Zhang and P Zhang. Soy isoflavone: The multipurpose phytochemical (Review). Biomed. Rep. 2013; 1, 697-701.

[7] AOAC. Official Methods of Analysis of AOAC International. 20 $0^{\text {th }}$ eds. Association of Official Analytical Chemists International, Maryland, USA, 2016.

[8] AOAC. Official Methods of Analysis: AOAC Official Method. Association of Official Analytical Chemists, Washington DC, USA, 2010. 
http://wjst.wu.ac.th

[9] PJV Soest, JB Robertson and BA Lewis. Methods of dietary fiber, neutral detergent fiber and nonstarch polysaccharides in relation to animal nutrition. J. Dairy Sci. 1991; 74, 3583-97.

[10] RE Burns. Method for estimation of tannin in grain sorghum. J. Agron. 1971; 63, 511-2.

[11] J Kubola and S Siriamornpun. Phytochemicals and antioxidant activity of different fruit fractions (peel, pulp, aril and seed) of Thai gac (Momordica cochinchinensis Spreng). Food Chem. 2011; 127, 1138-45.

[12] J Kubola, S Siriamornpun and N Meeso. Phytochemicals, vitamin C and sugar content of Thai wild fruits. Food Chem. 2011; 126, 972-81.

[13] MKA Devi, M Gondi, G Sakthivelu, P Giridhar, T Rajasekaran and R Gokare. Functional attributes of soybean seeds and products, with reference to isoflavone content and antioxidant activity. Food Chem. 2009; 114, 771-6.

[14] B Thiele, N Stein, M Oldiges and D Hofmann. Direct analysis of underivatized amino acids in plant extracts by LC-MS-MS. In: MA Alterman and P Hunziker (Eds.). Amino Acid Analysis: Methods and Protocols, Methods in Molecular Biology. NJ Totowa, Humana Press, 2012, p. 317-28.

[15] MS Nimbalkar, SR Pai, NV Pawar, D Oulkar and GB Dixit. Free amino acid profiling in grain Amaranth using LC-MS/MS. Food Chem. 2012; 134, 2565-9.

[16] P Wanyo, N Kaewseejan, N Meeso and S Siriamornpun. Bioactive compounds and antioxidant properties of different solvent extracts derived from Thai rice by-products. Appl. Biol. Chem. 2016; 59, 373-84.

[17] R Core Team. R: A language and environment for statistical computing. R Foundation for Statistical Computing, Available at: http://www.R-project.org, accessed April 2020.

[18] Coop de France. Déshydratation. Tout sur la luzerne, Available at: http: //www. tout-sur-laluzerne.com/intro-tout-sur-la-luzerne.html, accessed March 2020.

[19] D Undersander, MH Hall, P Vassalotti and D Cosgrove. Alfalfa germination \& growth. National Alfalfa \& Forage Alliance, 2011, p. 1-18.

[20] SL Ishaq, MM Lachman, BA Wenner, A Baeza, M Butler, E Gates, S Olivo, JB Geddes, P Hatfield and CJ Yeoman. Pelleted-hay alfalfa feed increases sheep wether weight gain and rumen bacterial richness over loose-hay alfalfa feed. PLoS One 2019; 14, e0215797.

[21] JB Harborne. Twenty-five years of chemical ecology. Nat. Prod. Rep. 2001; 18, 361-79.

[22] M Gxasheka, LT Tyasi, N Qin and ZC Lyu. An overview of tannins rich plants as alternative supplementation on ruminant animals: A review. Int. J. Agric. Res. 2015; 3, 343-9.

[23] HPS Makkar, M Bluemmel and K Becker. In vitro effects of and interactions between tannins and saponins and fate of tannins in the rumen. J. Sci. Food Agric. 1995; 69, 481-93.

[24] B Min, TN Barry, G Attwood and WC McNabb. The effect of condensed tannins on the nutrition and health of ruminants fed fresh temperature forages: A review. Anim. Feed Sci. Technol. 2003; 106, 3-19.

[25] IA Kagan, BM Goff and MD Flythe. Soluble phenolic compounds in different cultivars of red clover and alfalfa, and their implication for protection against proteolysis and ammonia production in ruminants. Nat. Prod. Commun. 2015; 10, $1263-7$.

[26] K Dajanta and H Rongkom. Effects of drying temperature on the isoflavone content and antioxidant capacity of fermented soybean (Thua Nao) (in Thai). KKU Sci. J. 2017; 45, 138-50.

[27] R Wang, SW Liu, XW Xin, S Chen, GX Peng, YJ Su and ZK Song. Phenolic acids contents and allelopathic potential of 10-cultivars of alfalfa and their bioactivity. Allelopathy J. 2017; 40, 63-70.

[28] L Sun, XZ Ren, GT Ge, XC Feng, Y Liu, ML Hou and YS Jia. Allelopathic effect of aqueous extracts from alfalfa stem and leaf on two gramineous forages (in Chinese). J. Northwest A\&F Univ. (Nat. Sci. Ed.). 2013; 41, 49-53.

[29] T Kobayashi, T Nakata and T Kuzumaki. Effect of flavonoids on cell cycle progression in prostate cancer cells. Cancer Lett. 2002; 176, 17-23.

[30] P Sunita and SP Pattanayak. Phytoestrogens in postmenopausal indications: A theoretical perspective. Pharmacogn. Rev. 2011; 5, 41-7.

[31] IMCM Rietjens, J Louisse and K Beekmann. The potential health effects of dietary phytoestrogens. Br. J. Pharmacol. 2017; 174, 1263-80. 
http://wjst.wu.ac.th

[32] AL Ososki and EJ Kennelly. Phytoestrogens: A review of the present state of research. Phytother. Res. 2003; 17, 845-69.

[33] H Steinshamn, S Purup, E Thuen and J Hansen-Moller. Effects of clover-grass silages and concentrate supplementation on the content of phytoestrogens in dairy cow milk. J. Dairy Sci. 2008; 91, 2715-25.

[34] EA Mustonen, M Tuori, I Saastamoinen, J Taponen, K Wahala, H Saloniemi and A Vanhatalo. Equol in milk of dairy cows is derived from forage legumes such as red clover. Br. J. Nutr. 2009; 102, 1552-6.

[35] CS Kwak, MS Lee and SC Park. Higher antioxidant properties of Chungkookjang, a fermented soybean paste, may be due to increased aglycone and malonylglycoside isoflavone during fermentation. Nutr. Res. 2007; 27, 719-27.

[36] D Wang, LJ Wang, FX Zhu, JY Zhu, XD Chen, L Zou, M Saito and LT Li. In vitro and in vivo studies on the antioxidant activities of the aqueous extracts of Douchi (a traditional Chinese saltfermented soybean food). Food Chem. 2008; 107, 1421-8.

[37] JY Je, ZJ Qian, HG Byun and SK Kim. Purification and characterization of an antioxidant peptide obtained from tuna backbone protein by enzymatic hydrolysis. Process Biochem. 2007; 42, 840-6.

[38] A Moawad and E Amin. Comparative antioxidant activity and volatile oil composition of leaves and fruits of Thuja orientalis growing in Egypt. Walailak J. Sci. Technol. 2019; 16, 823-30.

[39] A An, M Sunanda, B Shubhangi, A Jain and M Manisha. Pharmacognostic, phytochemical, and pharmacological investigation on bark of Thuja orientalis Linn (Cupressaceae). J. Pharmacogn. Phytochem. 2016; 5, 111-3. 